\title{
Prevenção e Atendimento Inicial do Trauma e Doenças Cardiovasculares: um Programa de Ensino
}

\author{
Prevention and Life Support in Case of \\ Trauma and Cardiovascular Diseases: an \\ Educational Program
}

Fernando Bueno Pereira Leitão ${ }^{I}$ Monica Caetano de Sousa ${ }^{I I}$

Dario Birolini ${ }^{I}$ Joaquim Edson Vieira

PALAVRAS-CHAVE:

- Primeiros Socorros;

- Ressuscitação Cardiopulmonar;

- Educação Médica.

KEY WORDS:

- First Aid;

- Cardiopulmonary Resuscitation;

- Education, Medical.

Recebido em: 31/08/2007

Aprovado em: 14/12/2007
- Universidade de São Paulo. Faculdade de Medicina, São Paulo, Brasil

"Centro de Estudos e Pesquisas Dr. João Amorim, São Paulo, Brasil.
A Faculdade de Medicina da Universidade de São Paulo, ao cumprir as prerrogativas da universidade quanto ao ensino, pesquisa e prestação de serviços à comunidade, tem desenvolvido programas direcionados à graduação e à coletividade, abrangendo diversos aspectos do trauma e das doenças cardiovasculares. Respeitando protocolos internacionais, cursos teórico-práticos são organizados e ministrados por instrutores reconhecidos pela American Heart Association e American College of Surgeons. A comparação entre pré e pós-testes demonstrou resultado melhor quando os alunos eram profissionais da área da saúde, o que foi atribuído a seu melhor preparo em relação à comunidade leiga. Entretanto, como a finalidade era a capacitação de todos, profissionais da saúde ou não, uma reavaliação da metodologia tornou-se necessária, salientando-se como principal preocupação uma duração maior das atividades práticas e maior possibilidade de discussões.

\section{ABSTRACT}

Pursuing its mission of teaching, research and delivery of services to the community, the University of São Paulo Medical School has developed programs directed not only to its students but also to the community at large. One of these programs addresses first aid in case of trauma and cardiovascular diseases. Following international guidelines recognized by the American Heart Association and the American College of Surgeons, the institution offers theoretical and practical courses organized and held by certified instructors. A comparison of the tests made before and after the course showed that the health professionals achieved better results, probably as a result of their previous professional training. However, as the main goal of the course was providing first-aid training to the lay community, the used methodology was reviewed and more attention was given to the practical activities and space for discussions 


\section{INTRODUÇÃO}

O trauma ${ }^{1,2} \mathrm{e}$ as doenças cardiovasculares $(\mathrm{DCVs})^{3}$ integram as principais causas de morte em todos os continentes, sendo que na América do Sul o Brasil vem apresentando uma casuística preocupante no decorrer destas duas últimas décadas ${ }^{1}$.

Atualmente reconhecido como doença, o trauma atingiu o adulto e a criança em todas as faixas etárias, sem distinção de raça ou condição socioeconômica ${ }^{1}$. O prejuízo dele decorrente pode ser representado pelo indicador "Anos Potenciais de Vida Perdidos" (APVP), que se refere à perda de vidas produtivas. As principais conseqüências são a piora da qualidade de vida, inclusive dos familiares e/ou responsáveis pelas vítimas, os custos econômicos e a sobrecarga da Previdência Social $^{4}$. Em 1997, por exemplo, a violência apresentava um custo de 1,9\% do PIB ${ }^{4}$ ! Estas mesmas considerações relacionadas ao APVP se aplicam aos casos de DCVs e suas complicações, como o infarto do miocárdio (IM) e os acidentes vasculares cerebrais (AVCs) - tanto o isquêmico (AVCI) como o hemorrágico $(\mathrm{AVCH})$-, por acarretarem prejuízo das atividades profissionais e, até, o óbito ${ }^{3,5}$.

Considerando-se também que estas ocorrências têm como local de origem o ambiente doméstico e outras áreas públicas e privadas, é fundamental destacar a importância de medidas preventivas e assistenciais por parte de profissionais das áreas da saúde ${ }^{6}$ e da comunidade leiga ${ }^{7}$.

Ainda quanto ao trauma, a prevenção deve ser dirigida principalmente às suas causas mais freqüentes, como as quedas de lajes ou em solos derrapantes, irregulares ou revestidos com tapetes escorregadios, a falta de corrimão nas escadas e banheiros, e o uso de calçados inadequados, comumente responsáveis por quedas em idosos. Outro aspecto a considerar é a obstrução de vias aéreas por corpos estranhos (Ovaces), conseqüente a volume excessivo de alimento e objetos colocados na boca e à submersão em lagoas, mar ou piscina ${ }^{3}$. Aliás, a Ovace é a principal causa de parada cardíaca na criança ${ }^{3}$.

O uso de álcool e líquidos quentes, os fogos de artifício e a exposição inadequada à luz solar têm sido responsáveis por queimaduras de primeiro, segundo e terceiro graus, e por isto também merecem especial atenção.

Quanto às agressões, geralmente conseqüentes à ingestão de álcool e drogas ilícitas, é importante destacar a medida governamental, em discussão, referente à proibição de armas de fogo, o combate às drogas ilícitas e as ocorrências do trânsito, responsáveis por diversos traumas e, mesmo, pelo óbito. Erroneamente classificadas como acidentes, as ocorrências do trânsito são, na realidade, produto da desatenção de motoristas e pedestres, e por isto salientamos a necessidade de respeito ao Código Nacional de Trânsito como atitude prioritária para evitá-las.
Quanto à prevenção das DCVs, é fundamental o combate ao fumo, à obesidade e à vida sedentária, bem como a necessidade do controle sistemático da pressão arterial e das condições clínicas ${ }^{3}$.

A par da destacada importância da prevenção, o atendimento imediato e correto - isto é, os primeiros socorros prestados a um doente traumatizado ou portador de quadro clínico condizente com risco de vida iminente - é um fator fundamental para uma evolução clínica desejável ${ }^{2}$. Isto porque favorece as ações que se seguirão, por conta do atendimento especializado prestado pelo médico e enfermagem, principalmente quando adotados os protocolos organizados pelo American College of Surgeons e pela Sociedade Brasileira de Atendimento Integrado ao Traumatizado (SBAIT).

Quanto aos casos de DCVs, o atendimento inicial deve obedecer aos protocolos definidos pela American Heart Association (AHA) e adotados pela Sociedade Brasileira de Cardiologia quanto ao Suporte Básico de Vida (BLS/SBV), com prioridade para solicitação de socorro, manobras iniciais de permeabilização das vias aéreas, ventilação pulmonar, circulação e desfibrilação, Assim, a continuidade do atendimento baseado no protocolo do Suporte Avançado de Vida (SAV) terá, também, a perspectiva de uma evolução mais favorável ${ }^{3}$.

Estes aspectos, que a partir de 1992 foram desenvolvidos no Curso de Reanimação vinculado à Disciplina de Anestesiologia da $\mathrm{FMUSP}^{6}$, passaram a integrar o Programa de Atenção a Situações de Risco e Atendimento Inicial da Parada Cardíaca (PC), da Disciplina de Cirurgia do Trauma da FMUSP. Seu objetivo é salientar a importância da prevenção ${ }^{8}$ e do treinamento dos profissionais da saúde e da comunidade leiga ${ }^{8}$ frente às situações de risco decorrentes do trauma ou condições clínicas.

A finalidade deste trabalho é avaliar o resultado da aplicação do programa.

\section{METODOLOGIA}

Os cursos abordaram a prevenção e o atendimento inicial do trauma e da parada cardíaca. Foram coordenados por um médico e tiveram a participação de médico, enfermeira e acadêmicos de Medicina e de Enfermagem, todos eles portadores do título de instrutor dos Cursos de Suporte Básico de Vida (Basic Life Support) pela AHA. Outros acadêmicos, não portadores do título, atuaram como monitores, colaborando com o treinamento prático.

Os cursos foram desenvolvidos para dois grupos: um, o GP, constou de 47 profissionais da área da saúde integrados por acadêmicos de Medicina de uma faculdade privada do Estado de São Paulo e médicos, enfermeiros e auxiliares de 
enfermagem de um hospital público da cidade de São Paulo; o outro, GL, com 59 integrantes da comunidade leiga, foi constituído de professores de primeiro e segundo graus, de funcionários de hospital público da cidade de São Paulo e de uma unidade da USP, de engenheiros e de escotistas.

A carga horária de cada um dos cursos foi de oito horas, iniciando-se pela apresentação dos alunos, dos instrutores e dos monitores. A seguir, foi distribuído um questionário (préteste) com 20 questões de múltipla escolha com apenas uma resposta correta e não contendo resposta "não sei". O mesmo questionário foi apresentado no final do curso (pós-teste) para avaliação imediata do aproveitamento. Ao final de cada teste, com duração de até 20 minutos, os questionários foram recolhidos a fim de não serem divulgados para outros eventuais interessados.

Após a realização do pré-teste foi ministrada aula teórica com uma hora de duração, abrangendo a prevenção do trauma e das DCVs e os aspectos do atendimento inicial a eventuais vítimas, bem como a importância de um atendimento continuado, conforme a Corrente da Sobrevivência.

Para melhor aproveitamento das atividades práticas, cada um dos grupos foi subdividido em outros, de até dez alunos cada.

O material utilizado para o treinamento de primeiros socorros constou de talas para imobilização, colar cervical, gaze e ataduras, manequins e participação dos alunos e instrutores como manequins vivos. Foram salientadas as seguintes condutas:

- compressão de ferimento para estancar sangramento;

- imobilização de membros supostamente fraturados e/ou luxados;

- limpeza de ferimentos com água e sabão;

- água fria corrente em região com queimadura de primeiro e segundo graus;

- imobilização de coluna cervical com colar ou outro artefato quando houver um ou mais traumatismos acima da clavícula ou mais que um traumatismo abaixo da clavícula;

- proteção de globo ocular e encaminhamento imediato ao oftalmologista quando houver qualquer tipo de traumatismo;

- proteção e posicionamento em decúbito lateral de vítimas com crise convulsiva ou nível de consciência rebaixado e respiração espontânea;

- importância da prevenção.

Para o treinamento do SBV foram utilizados manequins de criança, de jovem e de adulto (Laerdal) e desfibrilador externo automático (DEA), respeitando o protocolo da AHA.
Um manequim vivo foi a referência para simular a desobstrução de vias aéreas. A seqüência do atendimento constou de estímulo à tosse e da manobra de Heimlich no caso de persistência da obstrução, que não era realizada a fim de evitar eventual traumatismo torácico ou abdominal. $\mathrm{O}$ treinamento da desobstrução da criança foi feito com o manequim, iniciando-se por cinco pancadas firmes na região interescapular, seguidas de cinco compressões no terço inferior do externo, sucessivamente, por até um minuto. Após este tempo, com a persistência da obstrução, o socorro era solicitado, e as manobras continuavam. Ao simular uma PC, impunha-se a necessidade do SBV, com a possibilidade de observação da cavidade bucal; se o corpo estranho fosse visível, poderia ser retirado.

$\mathrm{O}$ treinamento com os manequins de adulto, jovem e criança foi realizado em rodízio, com os subgrupos, sendo um instrutor responsável por até dez alunos.

Durante todo o treinamento, o conteúdo da aula teórica foi reafirmado, e as eventuais dúvidas ou dificuldades foram resolvidas de imediato, sem preocupação com a carga horária. Os instrutores avaliaram o desempenho dos alunos quanto à presteza e correção de cada uma das manobras realizadas.

Após a realização do pós-teste, foi explicada a resposta correta de cada uma das questões. Em seguida, foi aberta uma discussão sobre o curso, ouvindo-se a opinião dos alunos e dos instrutores.

Os testes foram classificados pela avaliação de Chang-teh Fan. A distribuição das respostas foi avaliada pelo teste Kolmogov-Smirnov e os momentos pré e pós pelo teste de Wilcoxon (SigmaStat v3.11, 2004).

\section{RESULTADOS}

Todas as questões foram consideradas fáceis e nenhum teste foi excluído na análise comparativa. Os pré-testes dos dois grupos (Saúde e Leigos) apresentaram distribuição normal (Kolmogorov-Smirnov), enquanto os pós-testes não apresentaram a mesma distribuição (Tabela 1). A comparação pré versus pós foi realizada pelo teste de Wilcoxon. As médias de acertos foram superiores para os dois grupos, quando comparados os resultados pré e pós.

O percentual de acertos desejado para os dois testes foi definido como sendo de pelo menos $90 \%$. Verificou-se que ele não foi alcançado no pré-teste em nenhum dos grupos, ao passo que no pós-teste foi alcançado por 30 dos 46 profissionais do GP e por 21 dos 59 leigos (GL).

A crítica dos alunos foi direcionada à duração do treinamento, considerada insuficiente pela maioria deles.

Os instrutores obedeceram a todas as diretrizes do protocolo e constataram que a permeabilização das vias aéreas e a 
TABELA 1

Percentual de acerto. Médias \pm DP e medianas para os grupos Saúde e Leigos, pré e pós-treinamento em SBV

\begin{tabular}{|c|c|c|c|c|}
\hline & Saúde Pré (46) & Saúde Pós (46) & Leigos Pré (59) & Leigos Pós (59) \\
\hline Média \pm DP & $63,48 \pm 17,22$ & & $56,78 \pm 10,90$ & \\
\hline Mediana (25 - 75) & $65(50-80)$ & $90(85$ - 95) & $55(50-65)$ & $85(75-90)$ \\
\hline Wilcoxon & \multicolumn{2}{|c|}{$\mathrm{P}<0,001$} & \multicolumn{2}{|c|}{$\mathrm{P}<0,001$} \\
\hline
\end{tabular}

respiração boca a boca foram os procedimentos considerados mais difíceis e que exigiram maior atenção. Mereceu destaque a capacitação de acadêmicos como instrutores dos cursos de SBV, o que se reflete como estímulo para outros.

Todos os alunos entenderam a importância dos protocolos.

\section{COMENTÁRIOS}

Os casos de trauma e DCVs, responsáveis por grande incidência de óbitos, têm motivado estudos que abrangem suas causas, prevenção, atendimento inicial e continuado ao doente e as mais diversas conseqüências, como seqüelas, óbito e suas repercussões socioeconômicas ${ }^{1}$. Tais aspectos demonstram a responsabilidade da universidade ${ }^{6}$, de organizações não-governamentais e da própria comunidade quanto à conscientização sobre uma verdadeira catástrofe que atinge a população ${ }^{9}$, mas que pode ser enfrentada e, pelo menos, diminuída. Neste sentido, deve-se estimular e desenvolver atitudes relacionadas fundamentalmente à prevenção ${ }^{10} \mathrm{e}$ adoção de protocolos muito bem definidos.

Neste estudo, verificamos a importância de um trabalho que aborda tais aspectos, como o Programa de Atenção às Situações de Risco e Atendimento Inicial à Parada Cardíaca, da Disciplina de Cirurgia do Trauma da FMUSP, cuja principal característica é a sistemática do ensino direcionado a alunos de graduação de Medicina e Enfermagem. A elaboração de testes apresentados antes e após a realização de cursos de primeiros socorros e SBV permitiu avaliar a capacitação de todos os alunos. Embora, pelo método estatístico empregado, todas as questões tenham sido consideradas fáceis, surpreendeu-nos a ausência de acertos (90\% ou mais) no pré-teste, principalmente nos GL. Entendemos, então, que a distribuição prévia de uma apostila com o conteúdo dos cursos facilite o entendimento da matéria exposta na aula teórica e discutida durante o treinamento. Quanto ao pós-teste, o melhor aproveitamento do GP é compreensível e certamente decorre da formação científica e atividade profissional, mas supúnhamos que os resultados apresentassem maior índice de acertos.

No final dos cursos constatamos a validade do programa e salientamos a importância de sua constante avaliação, visando, quando for o caso, às alterações necessárias, sempre respeitando seu objetivo e o protocolo do SBV.
A participação de acadêmicos que se tornaram instrutores é um estímulo para que outros obtenham o mesmo título. Foi nítida a satisfação que demonstraram ao se sentirem capacitados para o ensino de profissionais da saúde e da comunidade leiga, criando-se uma perspectiva a ser analisada por responsáveis por cursos de graduação em escolas médicas e de Enfermagem.

$\mathrm{O}$ interesse demonstrado pelos alunos durante a realização dos cursos vem ao encontro, principalmente, da prática do treinamento ${ }^{8}$. Embora os alunos tenham criticado a pequena duração dos cursos, reafirmamos que, por serem cursos de treinamento, eles devem simular situações que exigem presteza do aluno, que irá atuar como socorrista. Essa duração, entretanto, não deve prejudicar o detalhamento das manobras, mesmo que seja preciso repeti-las. É responsabilidade do instrutor avaliar o desempenho de cada aluno e, se for o caso, possibilitar a oportunidade de aperfeiçoamento. A experiência dos instrutores comprovou que a duração dos treinamentos, por ser relativamente curta, influencia a exigência de maior atenção aos assuntos abordados. O melhor resultado apresentado pelo pós-teste confirma esta observação, pois todos se mantiveram muito atentos ao desenvolvimento da programação.

Um significativo destaque do programa foi o número de interessados, o que reafirma a necessidade e importância da prevenção, primeiros socorros e treinamento em SBV como fatores de grande relevância quanto ao cuidado geral e universal de saúde, principalmente por abordar situações de risco, de ordem clínica e do trauma.

A participação de alunos da FMUSP e de Enfermagem como instrutores e como monitores em treinamento para obtenção do título de instrutores de SBV pela AHA apresentou duas vantagens: o estímulo ao seu próprio desenvolvimento e a colaboração prestada no desempenho das atividades práticas.

\section{CONCLUSÕES}

O programa despertou o interesse dos acadêmicos que se tornaram instrutores e participaram de cursos destinados à formação de recursos humanos nas áreas do trauma e doenças cardiovasculares.

Profissionais da área da saúde e da comunidade leiga entenderam a necessidade de prevenção de situações de agravo 
à saúde e da capacitação no atendimento inicial de eventuais vítimas.

Respeitando a carga horária de oito horas, é importante a revisão continuada da programação, tendo em vista maior interação com os alunos.

Deve-se dar maior ênfase ao treinamento principalmente das ações de permeabilização das vias aéreas e respiração boca a boca quando forem constatadas dificuldades em sua realização.

As informações teóricas devem se referir também a detalhes que possam ser obtidos em outros momentos e/ou por consulta a trabalhos específicos, mas sem prejuízo do treinamento prático.

O programa deverá se manter atualizado, obedecendo a protocolos definidos pelas entidades por eles responsáveis.

\section{AGRADECIMENTOS}

Os autores agradecem a contribuição de Aparecido Nakano Martins, Daniel Cordovani, Eduardo D'Alessandro, Ilana Sebbag, Johnny Leandro Conduta Borda Aldunate, Juan Jose Conceição Bomfim, Ligia Almeida Ferreira, Lincoln Saito Millan, Marcelo Cristiano Rocha, Mauricio Dener Cordeiro, Marcelo El Khouri, Rolison Bravo Lellis e Sérgio Nobuo Konno.

\section{REFERÊNCIAS}

1. Melo Jorge MHP, Gawryszewski VP, Latorre RDO. Rev. Saúde Pública 1997; 31 (4 Supl): 5-25.

2. Suporte avançado de vida no trauma para médicos: manual do curso para alunos. 6. ed. Chicago: American College of Surgeons; 1997.

3. Stapleton ER, ed. SBV para provedores de saúde. Dallas: American Heart Association, Rio de Janeiro: Fundação Interamericana do Coração; 2002.
4. Iunes R. Impacto econômico das causas externas no Brasil: um esforço de mensuração. Rev. Saúde Pública 1997; 31(4 Supl.): 38-46.

5. Cummins RO, ed. Advanced cardiac life support. Dallas: American Heart Association; 1997.

6. Leitão FBP, Amaral RVG, Birolini D. Ensino da reanimação e medicina intensiva. Rev. Hosp. Clin. Fac. Med. S.Paulo 1992; 47(5): 252-256.

7. Graziano KV, Gatto MAF, Silva JA, Leitão FBP. Fatores limitantes do socorrista na continuidade da ressuscitação cardiopulmonar em manequim tipo adulto, Panam. J. Trauma 1995; 5 (11): 146.

8. Steiman M, Steiman E, Ricci J, Koleski F, Lopes Jr. AG, Silva L, Cruz Jr. RJC, Patricio L, Poggetti RS. Interesse dos residentes de cirurgia em trauma. Panam. J. Trauma 1995; 5 (XI): 136.

9. Amaral VLAR, Mantovani M. Aspectos psicossociais de pacientes traumatizados por arma branca ou arma de fogo. Panam. J. Trauma 1996; 5 (11): 129.

10. Leitão FBP. Prevenção de acidentes de trânsito: modelo usado pela Organização Pan-Americana de Saúde. Rev. Saúde Pública, 1997; 31 (Supl.): 47-50.

\section{CONFLITOS DE INTERESSE}

Declarou não haver.

\section{ENDEREÇO PARA CORRESPONDÊNCIA}

Fernando Bueno Pereira Leitão

Faculdade de Medicina da Universidade de São Paulo Rua Prof. Horacio Berlinck, 71

05505-040 - São Paulo - SP

E-mail: fernandopleitao@yahoo.com.br 